\title{
A New Nonlinear H-infinity Feedback Control Approach to the Problem of Autonomous Robot Navigation
}

\author{
G. Rigatos ${ }^{1} \cdot$ P. Siano ${ }^{2}$
}

Received: 8 December 2014 / Revised: 27 April 2015 / Accepted: 19 July 2015 / Published online: 16 September 2015

(C) Springer Science+Business Media Singapore 2015

\begin{abstract}
This research work introduces a new control method for feedback control of nonlinear dynamical systems with application example the problem of trajectory tracking by an autonomous robotic vehicle. The control method consists of a repetitive solution of an H-infinity control problem for the mobile robot, that makes use of a locally linearized model of the robot and takes place at each iteration of the control algorithm. The vehicle's model is locally linearized round its current position through the computation of the associated Jacobian matrices. Using the linearized model of the vehicle an H-infinity feedback control law is computed. The known robustness features of $\mathrm{H}$-infinity control enable to compensate for the errors of the approximative linearization, as well as to eliminate the effects of external perturbations. The efficiency of the proposed control scheme is shown analytically and is confirmed through simulation experiments. The method can be applied to a wide class of nonlinear dynamical systems.
\end{abstract}

Keywords Nonlinear H-infinity feedback control · Robust control $\cdot$ Autonomous robot $\cdot$ Navigation

\footnotetext{
G. Rigatos

grigat@ieee.org

P. Siano

psiano@unisa.it

1 Unit of Industrial Automation, Industrial Systems Institute, 26504 Rion Patras, Greece

2 Department of Industrial Engineering, University of Salerno, 84084 Fisciano, Italy
}

\section{Introduction}

Nonlinear and embedded control and autonomous navigation of robotic vehicles is of primary importance for the automotive industry. By succeeding motion control of the vehicle, safety in driving can be improved while other several practical problems, such as lane keeping and maneuvering or parallel parking can be solved [1-8]. Up to now several research results have been developed to enable the steering control and autonomous navigation of vehicles. The developed approaches are based on nonlinear control, such as differential geometry and differential flatness theory approaches as well as on Lyapunov stability methods [9-15]. In this paper a new solution to the problem of autonomous vehicle navigation is given, using a linearization scheme together with $H_{\infty}$ robust control theory [16].

The kinematic model of a unicycle robotic vehicle is considered as a case study, however the proposed approach can be also applied to other types of vehicles (such as four wheel vehicles, heavy duty vehicles with implements, etc.). Actually the paper proposes the application of an approximate linearization scheme for the kinematic model of the unicycle robotic vehicle that is based on Taylor series expansion round the vehicle's current position. To perform this linearization the computation of Jacobian matrices is needed while the induced linearization error terms are treated as disturbances. For the linearized equivalent of the robot's model an $H_{\infty}$ feedback control scheme is developed. The formulation of the $H_{\infty}$ control problem is based on the minimization of a quadratic cost function that comprises both the disturbance and the control input effects. The disturbance tries to maximize the cost function while the control signal tries to minimize it, within a mini-max differential game.

Comparing to nonlinear feedback control approaches which are based on exact feedback linearization (as the ones 
based on differential flatness theory and analyzed in [16-18]) the proposed $H_{\infty}$ control scheme is assessed as follows: (i) it uses an approximative linearization approach of the system's dynamic or kinematic model which does not follow the elaborated transformations (diffeomorphisms) of the exact linearization methods, (ii) it introduces additional disturbance error which is due to the approximative linearization of the system dynamics coming from the application of Taylor series expansion, (iii) it requires the computation of Jacobian matrices, which in the case of systems of high dimensionality can be also a cumbersome procedure (see industrial robotic manipulators), (iv) unlike exact feedback linearization, the $H_{\infty}$ control term has to compensate not only for modelling uncertainties and external disturbances but needs also to annihilate the effects of the cumulative linearization error, and (v) the $H_{\infty}$ control approach follows optimal control methods for the computation of the control signal, however unlike exact feedback linearization control it requires the solution of Riccati equations which for systems of high dimensionality can be again a cumbersome procedure.

The structure of the paper is as follows: in "Linearization of the Robot's Kinematic Model" section the approach for approximate linearization of the vehicle's kinematic model is explained. In "The Nonlinear H-infinity Control" section the formulation of the $H_{\infty}$ nonlinear control problem for the linearized equivalent of the system is provided. In "Lyapunov Stability Analysis" section Lyapunov stability analysis for the developed control loop is given. In "Simulation Tests" section simulation tests are carried out to assess the performance of the feedback control loop. Finally, in "Conclusions" section concluding remarks are stated.

\section{Linearization of the Robot's Kinematic Model}

\section{Major Approaches in Control of Nonlinear Dynamical Systems}

Motion control of unmanned vehicles is a nonlinear control problem. One can distinguish three main approaches in the design of nonlinear control systems: (i) control and filtering based on global linearization methods, (ii) control and filtering based on asymptotic linearization methods, and (iii) Lyapunov methods.

As far as approach (i) is concerned, that is methods of global linearization, one can classify there methods for the transformation of nonlinear vehicles dynamics into equivalent linear state space form. For the linear equivalent forms of the vehicles dynamics one can design feedback controllers and can solve the problem of state estimation (filtering). In this area one can consider methods based on differential flatness theory and methods based on Lie algebra. These approaches avoid approximation errors in modelling and arrive at controllers of increased precision and robustness. In this area one can also note research on a new nonlinear filtering method (derivative-free nonlinear Kalman filter) which is more precise and computationally faster than other nonlinear estimation approaches.

As far as approach (ii) is concerned, that is methods of asymptotic linearization, it is anticipated to continue research on robust and adaptive control with the use of a decomposition of the vehicles dynamics into a set of linear local models. One can pursue solutions to the problem of nonlinear control, based on local linear models (round linearization points). For such systems one can select the parameters of the local controllers by following linear feedback controller design methods. These controllers succeed asymptotically, that is in the course of time, the compensation of the nonlinear system dynamics and the stabilization of the feedback control loops. In this thematic area one can note research on a new nonlinear H-infinity control method, which is based on the local and approximative linearization of the vehicles dynamics and which makes use of the computation of Jacobian matrices.

As far as approach (iii) is concerned, that is Lyapunov theory-based nonlinear control methods one comes against problems of minimization of the Lyapunov functions so as to compute control signals for nonlinear vehicle dynamics. For the development of Lyapunov-type controllers one can either exploit a model of the vehicles dynamics, or can avoid completely the use of such a model as in the case of adaptive control. In the later case, the vehicles dynamics is completely unknown and can be approximated by adaptive algorithms which are suitably designed so as to assure the stability and robustness of the control loop.

The article's results serve the development of the aforementioned research direction (ii), that is nonlinear control based on approximate linearization methods.

\section{Linearization of the Mobile Robot Through Taylor Series Expansion}

A unicycle autonomous robotic vehicle is considered. Its kinematic model is given by

$$
\left(\begin{array}{c}
\dot{x} \\
\dot{y} \\
\dot{\theta}
\end{array}\right)=\left(\begin{array}{cc}
\cos (\theta) & 0 \\
\sin (\theta) & 0 \\
0 & 1
\end{array}\right) \cdot\left(\begin{array}{c}
v \\
\omega
\end{array}\right),
$$

where $x, y$ are the cartesian coordinates of the robot's center of gravity and $\theta$ is its orientation angle. Input $v$ is the vehicle's linear velocity and $\omega$ is the vehicle's angular velocity for rotations round its transversal axis. Considering linearization of the model round the current position of the robot and round the velocity value $v\left(t-T_{s}\right)$, where $T_{S}$ is the sampling period, 
the Jacobian matrices of the robotic model are:

$$
\begin{aligned}
A & =\left(\begin{array}{ccc}
0 & 0 & -v\left(t-T_{S}\right) \cdot \sin (\theta) \\
0 & 0 & v\left(t-T_{S}\right) \cdot \cos (\theta) \\
0 & 0 & 0
\end{array}\right), \\
B & =\left(\begin{array}{cc}
\cos (\theta) & 0 \\
\sin (\theta) & 0 \\
0 & 1
\end{array}\right) .
\end{aligned}
$$

The state vector of the robotic vehicle is denoted as $\mathbf{x}=$ $[\mathbf{x}, \mathbf{y}, \theta]^{\mathbf{T}}$ while the input vector is denoted as $u=[v, w]^{T}$. After linearization round its current position, the robot's kinematic model is written as

$\dot{x}=A x+B u+d_{1}$

Parameter $d_{1}$ stands for the linearization error in the robot's kinematic model appearing in Eq. (4). The desirable trajectory of the robot is denoted by $\mathbf{x}_{\mathbf{d}}=\left[\mathbf{x}_{\mathbf{d}}, \mathbf{y}_{\mathbf{d}}, \theta_{\mathbf{d}}\right]$. Tracking of this trajectory is succeeded after applying the control input $u^{*}$. At every time instant the control input $u^{*}$ is assumed to differ from the control input $u$ appearing in Eq. (4) by an amount equal to $\Delta u$, that is $u^{*}=u+\Delta u$

$\dot{x}_{d}=A x_{d}+B u^{*}+d_{2}$.

The dynamics of the controlled system described in Eq. (4) can be also written as

$\dot{x}=A x+B u+B u^{*}-B u^{*}+d_{1}$,

and by denoting $d_{3}=-B u^{*}+d_{1}$ as an aggregate disturbance term one obtains

$\dot{x}=A x+B u+B u^{*}+d_{3}$.

By subtracting Eq. (5) from Eq. (7) one has

$\dot{x}-\dot{x}_{d}=A\left(x-x_{d}\right)+B u+d_{3}-d_{2}$.

By denoting the tracking error as $e=x-x_{d}$ and the aggregate disturbance term as $\tilde{d}=d_{3}-d_{2}$, the tracking error dynamics becomes

$\dot{e}=A e+B u+\tilde{d}$.

The above linearized form of the robotic model can be efficiently controlled after applying an H-infinity feedback control scheme.

\section{The Nonlinear H-infinity Control}

\section{Mini-max Control and Disturbance Rejection}

The initial nonlinear system is assumed to be in the form

$\dot{x}=f(x, u) \quad x \in R^{n}, \quad u \in R^{m}$.

Linearization of the system (autonomous vehicle) is performed at each iteration of the control algorithm round its present operating point $\left(x^{*}, u^{*}\right)=\left(x(t), u\left(t-T_{s}\right)\right)$. The linearized equivalent of the system is described by

$\dot{x}=A x+B u+L \tilde{d} \quad x \in R^{n}, \quad u \in R^{m}, \quad \tilde{d} \in R^{q}$,

where matrices $A$ and $B$ are obtained from the computation of the Jacobians

$\begin{aligned} & A=\left.\left(\begin{array}{llll}\frac{\partial f_{1}}{\partial x_{1}} & \frac{\partial f_{1}}{\partial x_{2}} & \cdots & \frac{\partial f_{1}}{\partial x_{n}} \\ \frac{\partial f_{2}}{\partial x_{1}} & \frac{\partial f_{2}}{\partial x_{2}} & \cdots & \frac{\partial f_{2}}{\partial x_{n}} \\ \cdots & \cdots & \cdots & \cdots \\ \frac{\partial f_{n}}{\partial x_{1}} & \frac{\partial f_{n}}{\partial x_{2}} & \cdots & \frac{\partial f_{n}}{\partial x_{n}}\end{array}\right)\right|_{\left(x^{*}, u^{*}\right),} \\ & B=\left.\left(\begin{array}{lllll}\frac{\partial f_{1}}{\partial u_{1}} & \frac{\partial f_{1}}{\partial u_{2}} & \cdots & \frac{\partial f_{1}}{\partial u_{m}} \\ \frac{\partial f_{2}}{\partial u_{1}} & \frac{\partial f_{2}}{\partial u_{2}} & \cdots & \frac{\partial f_{2}}{\partial u_{m}} \\ \cdots & \cdots & \cdots & \cdots \\ \frac{\partial f_{n}}{\partial u_{1}} & \frac{\partial f_{n}}{\partial u_{2}} & \cdots & \frac{\partial f_{n}}{\partial u_{m}}\end{array}\right)\right|_{\left(x^{*}, u^{*}\right),}\end{aligned}$

and vector $\tilde{d}$ denotes disturbance terms due to linearization errors. The problem of disturbance rejection for the linearized model that is described by

$\dot{x}=A x+B u+L \tilde{d}$,

$y=C x$,

where $x \in R^{n}, u \in R^{m}, \tilde{d} \in R^{q}$ and $y \in R^{p}$, cannot be handled efficiently if the classical LQR control scheme is applied. This is because of the existence of the perturbation term $d$. The disturbance term $d$ apart from modeling (parametric) uncertainty and external perturbation terms can also represent noise terms of any distribution.

In the $H_{\infty}$ control approach, a feedback control scheme is designed for trajectory tracking by the system's state vector and simultaneous disturbance rejection, considering that the disturbance affects the system in the worst possible manner. The disturbances' effects are incorporated in the following quadratic cost function:

$$
\begin{aligned}
J(t)= & \frac{1}{2} \int_{0}^{T}\left[y^{T}(t) y(t)\right. \\
& \left.+r u^{T}(t) u(t)-\rho^{2} \tilde{d}^{T}(t) \tilde{d}(t)\right] d t, \quad r, \rho>0 .
\end{aligned}
$$


The significance of the negative sign in the cost function's term that is associated with the perturbation variable $\tilde{d}(t)$ is that the disturbance tries to maximize the cost function $J(t)$ while the control signal $u(t)$ tries to minimize it. The physical meaning of the relation given above is that the control signal and the disturbances compete to each other within a mini-max differential game. This problem of mini-max optimization can be written as

$\min _{u} \max _{\tilde{d}} J(u, \tilde{d})$.

The objective of the optimization procedure is to compute a control signal $u(t)$ which can compensate for the worst possible disturbance, that is externally imposed to the system. However, the solution to the mini-max optimization problem is directly related to the value of the parameter $\rho$. This means that there is an upper bound in the disturbances magnitude that can be annihilated by the control signal.

\section{H-infinity Feedback Control}

For the linearized system given by Eq. (14) the cost function of Eq. (15) is defined, where the coefficient $r$ determines the penalization of the control input and the weight coefficient $\rho$ determines the reward of the disturbances' effects.

It is assumed that (i) the energy that is transferred from the disturbances signal $d(t)$ is bounded, that is

$\int_{0}^{\infty} \tilde{d}^{T}(t) \tilde{d}(t) d t<\infty$

(ii) The matrices $[A B]$ and $[A L]$ are stabilizable, (iii) the matrix $[A C]$ is detectable. Then, the optimal feedback control law is given by

$u(t)=-K x(t)$,

with

$K=\frac{1}{r} B^{T} P$,

where $P$ is a positive semi-definite symmetric matrix which is obtained from the solution of the Riccati equation

$A^{T} P+P A+Q-P\left(\frac{1}{r} B B^{T}-\frac{1}{2 \rho^{2}} L L^{T}\right) P=0$,

where $Q$ is also a positive definite symmetric matrix. The worst case disturbance is given by

$\tilde{d}(t)=\frac{1}{\rho^{2}} L^{T} \operatorname{Px}(t)$.

\section{The Role of Riccati Equation Coefficients in $\boldsymbol{H}_{\infty}$ Control Robustness}

The parameter $\rho$ in Eq. (15), is an indication of the closedloop system robustness. If the values of $\rho>0$ are excessively decreased with respect to $r$, then the solution of the Riccati equation is no longer a positive definite matrix. Consequently there is a lower bound $\rho_{\min }$ of $\rho$ for which the $H_{\infty}$ control problem has a solution. The acceptable values of $\rho$ lie in the interval $\left[\rho_{\text {min }}, \infty\right)$. If $\rho_{\text {min }}$ is found and used in the design of the $H_{\infty}$ controller, then the closed-loop system will have increased robustness. Unlike this, if a value $\rho>\rho_{\text {min }}$ is used, then an admissible stabilizing $H_{\infty}$ controller will be derived but it will be a suboptimal one. The Hamiltonian matrix

$H=\left(\begin{array}{cc}A & -\left(\frac{1}{r} B B^{T}-\frac{1}{\rho^{2}} L L^{T}\right) \\ -Q & -A^{T}\end{array}\right)$,

provides a criterion for the existence of a solution of the Riccati equation Eq. (20). A necessary condition for the solution of the algebraic Riccati equation to be a positive semi-definite symmetric matrix is that $H$ has no imaginary eigenvalues [16].

\section{Lyapunov Stability Analysis}

Through Lyapunov stability analysis it will be shown that the proposed nonlinear control scheme assures $H_{\infty}$ tracking performance, and that in case of bounded disturbance terms asymptotic convergence to the reference setpoints is succeeded.

The tracking error dynamics for the robotic vehicle is written in the form

$\dot{e}=A e+B u+L \tilde{d}$,

where in the unicycle robot's application example $L=I \in R^{2}$ with $I$ being the identity matrix. The following Lyapunov equation is considered

$V=\frac{1}{2} e^{T} P e$,

where $e=x-x_{d}$ is the tracking error. By differentiating with respect to time one obtains

$$
\begin{aligned}
& \dot{V}=\frac{1}{2} \dot{e}^{T} P e+\frac{1}{2} e P \dot{e} \Rightarrow \\
& \dot{V}=\frac{1}{2}[A e+B u+L \tilde{d}]^{T} P+\frac{1}{2} e^{T} P[A e+B u+L \tilde{d}] \Rightarrow,
\end{aligned}
$$




$$
\begin{gathered}
\dot{V}=\frac{1}{2}\left[e^{T} A^{T}+u^{T} B^{T}+\tilde{d}^{T} L^{T}\right] P e \\
\quad+\frac{1}{2} e^{T} P[A e+B u+L \tilde{d}] \Rightarrow, \\
\dot{V}=\frac{1}{2} e^{T} A^{T} P e+\frac{1}{2} u^{T} B^{T} P e+\frac{1}{2} \tilde{d}^{T} L^{T} P e \\
+\frac{1}{2} e^{T} P A e+\frac{1}{2} e^{T} P B u+\frac{1}{2} e^{T} P L \tilde{d} .
\end{gathered}
$$

The previous equation is rewritten as

$$
\begin{aligned}
\dot{V}=\frac{1}{2} & e^{T}\left(A^{T} P+P A\right) e+\left(\frac{1}{2} u^{T} B^{T} P e+\frac{1}{2} e^{T} P B u\right) \\
& +\left(\frac{1}{2} \tilde{d}^{T} L^{T} P e+\frac{1}{2} e^{T} P L \tilde{d}\right) .
\end{aligned}
$$

Assumption For given positive definite matrix $Q$ and coefficients $r$ and $\rho$ there exists a positive definite matrix $P$, which is the solution of the following matrix equation

$$
A^{T} P+P A=-Q+P\left(\frac{1}{r} B B^{T}-\frac{1}{2 \rho^{2}} L L^{T}\right) P .
$$

Moreover, the following feedback control law is applied to the system

$u=-\frac{1}{r} B^{T} P e$.

By substituting Eqs. (29) and (30) one obtains

$$
\begin{aligned}
\dot{V}= & \frac{1}{2} e^{T}\left[-Q+P\left(\frac{1}{r} B B^{T}-\frac{1}{2 \rho^{2}} L L^{T}\right) P\right] e \\
& +e^{T} P B\left(-\frac{1}{r} B^{T} P e+e^{T} P L \tilde{d}\right) \Rightarrow, \\
\dot{V}=- & \frac{1}{2} e^{T} Q e+\left(\frac{1}{r} P B B^{T} P e-\frac{1}{2 \rho^{2}} e^{T} P L L^{T}\right) P e \\
& -\frac{1}{r} e^{T} P B B^{T} P e+e^{T} P L \tilde{d},
\end{aligned}
$$

which after intermediate operations gives

$\dot{V}=-\frac{1}{2} e^{T} Q e-\frac{1}{2 \rho^{2}} e^{T} P L L^{T} P e+e^{T} P L \tilde{d}$,

or, equivalently

$$
\begin{aligned}
\dot{V}=- & \frac{1}{2} e^{T} Q e-\frac{1}{2 \rho^{2}} e^{T} P L L^{T} P e \\
& +\frac{1}{2} e^{T} P L \tilde{d}+\frac{1}{2} \tilde{d}^{T} L^{T} P e .
\end{aligned}
$$

\section{Lemma The following inequality holds}

$$
\frac{1}{2} e^{T} L \tilde{d}+\frac{1}{2} \tilde{d} L^{T} P e-\frac{1}{2 \rho^{2}} e^{T} P L L^{T} P e \leq \frac{1}{2} \rho^{2} \tilde{d}^{T} \tilde{d} .
$$

Proof The binomial $\left(\rho \alpha-\frac{1}{\rho} b\right)^{2}$ is considered. Expanding the left part of the above inequality one gets

$\rho^{2} a^{2}+\frac{1}{\rho^{2}} b^{2}-2 a b \geq 0 \Rightarrow \frac{1}{2} \rho^{2} a^{2}+\frac{1}{2 \rho^{2}} b^{2}-a b \geq 0 \Rightarrow$

$a b-\frac{1}{2 \rho^{2}} b^{2} \leq \frac{1}{2} \rho^{2} a^{2} \Rightarrow \frac{1}{2} a b+\frac{1}{2} a b-\frac{1}{2 \rho^{2}} b^{2} \leq \frac{1}{2} \rho^{2} a^{2}$.

The following substitutions are carried out: $a=\tilde{d}$ and $b=e^{T} P L$ and the previous relation becomes

$\frac{1}{2} \tilde{d}^{T} L^{T} P e+\frac{1}{2} e^{T} P L \tilde{d}-\frac{1}{2 \rho^{2}} e^{T} P L L^{T} P e \leq \frac{1}{2} \rho^{2} \tilde{d}^{T} \tilde{d}$.

Equation (37) is substituted in Eq. (34) and the inequality is enforced, thus giving

$\dot{V} \leq-\frac{1}{2} e^{T} Q e+\frac{1}{2} \rho^{2} \tilde{d}^{T} \tilde{d}$.

Equation(38) shows that the $H_{\infty}$ tracking performance criterion is satisfied. The integration of $\dot{V}$ from 0 to $T$ gives

$$
\begin{gathered}
\int_{0}^{T} \dot{V}(t) d t \leq-\frac{1}{2} \int_{0}^{T}\|e\|_{Q}^{2} d t+\frac{1}{2} \rho^{2} \int_{0}^{T}\|\tilde{d}\|^{2} d t \Rightarrow \\
2 V(T)+\int_{0}^{T}\|e\|_{Q}^{2} d t \leq 2 V(0)+\rho^{2} \int_{0}^{T}\|\tilde{d}\|^{2} d t .
\end{gathered}
$$

Moreover, if there exists a positive constant $M_{d}>0$ such that

$\int_{0}^{\infty}\|\tilde{d}\|^{2} d t \leq M_{d}$

then one gets

$\int_{0}^{\infty}\|e\|_{Q}^{2} d t \leq 2 V(0)+\rho^{2} M_{d}$.

Thus, the integral $\int_{0}^{\infty}\|e\|_{Q}^{2} d t$ is bounded. Moreover, $V(T)$ is bounded and from the definition of the Lyapunov function $V$ in Eq. (24) it becomes clear that $e(t)$ will be also bounded since $e(t) \in \Omega_{e}=\left\{e \mid e^{T} P e \leq 2 V(0)+\rho^{2} M_{d}\right\}$.

According to the above and with the use of Barbalat's Lemma one obtains $\lim _{t \rightarrow \infty} e(t)=0$.

\section{Simulation Tests}

The performance of the proposed nonlinear $H_{\infty}$ control scheme is tested is two examples: (i) when the mobile robot tracks a reference trajectory, and (ii) when the mobile robot 
Fig. 1 a Plot of the circular $x-y$ trajectory followed by the mobile robot, and $\mathbf{b}$ convergence of the robot's state variables $x_{1}=x, x_{2}=y$ and $x_{3}=\theta$ to the associated reference setpoints

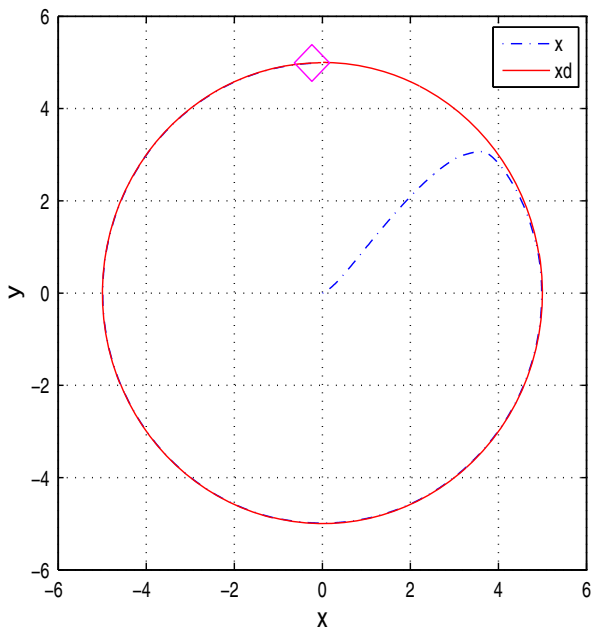

(a)

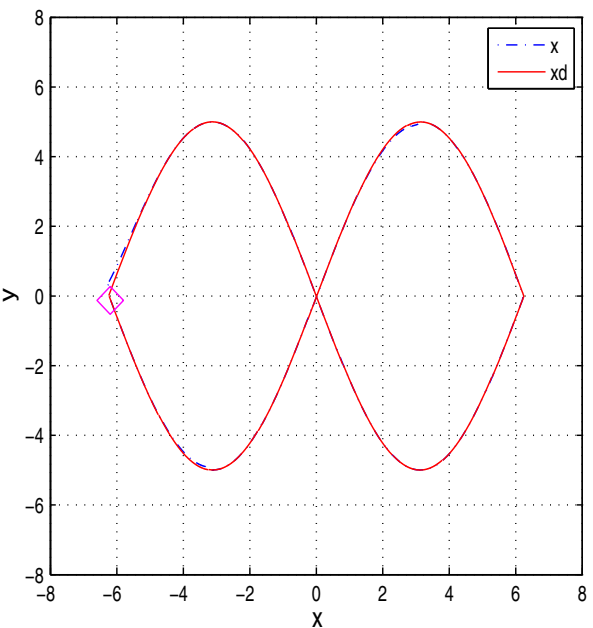

(a)
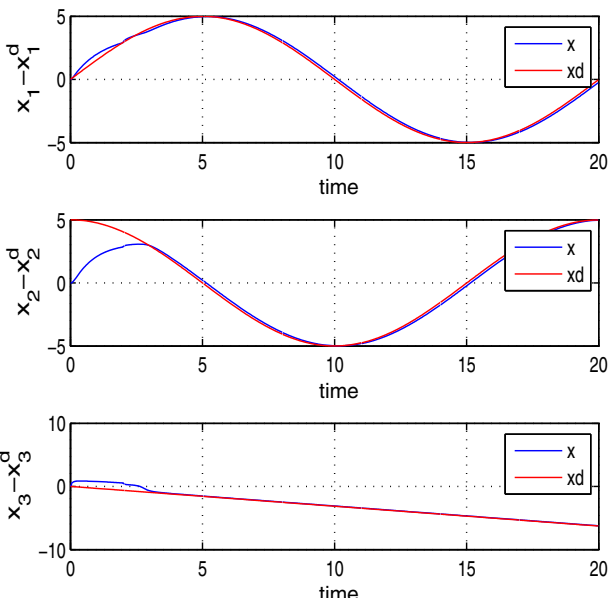

(b)
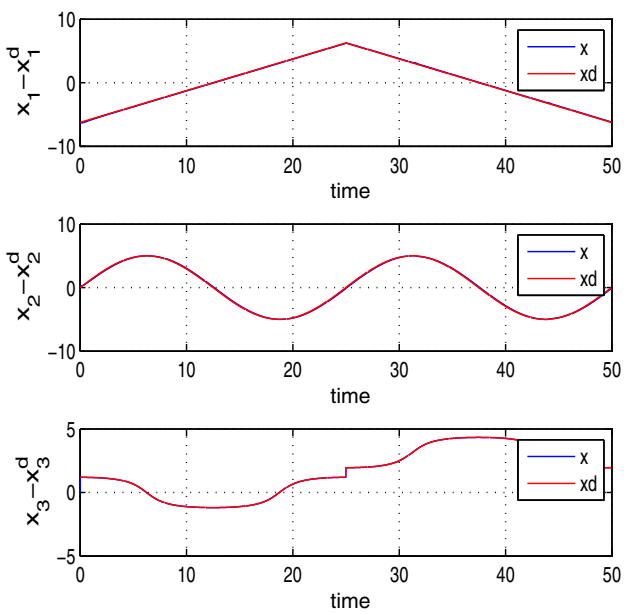

(b) performs the automated parallel parking task. In both cases the performance of the proposed controller was satisfactory, with minimum tracking error and fast convergence to the reference setpoints. In the computation of the reference path, the coordinates of the reference trajectory in the 2D-plane $\left(x_{d}, y_{d}\right)$ have been used, while the desirable steering angle has been computed by $\theta_{d}=\tan ^{-1}\left(\dot{y}_{d} / \dot{x}_{d}\right)$. The obtained results are depicted in Figs. 1, 2 and 3.

The tracking performance of the control method is shown in Tables 1 and 2. It can be observed that the tracking error for all state variables of the mobile robot was extremely small. Besides, in the simulation diagrams one can note the excellent transient performance of the control algorithm, which means that convergence to the reference path was succeeded in a smooth manner, while also avoiding overshoot and oscillations.

Remark 1 The errors and disturbances that affect the proposed control method are as follows: (i) linearization errors due to the truncation of higher order terms in the Taylor series expansion of the vehicle's nonlinear model, and (ii) external perturbations that may affect the vehicle's motion. H-infinity control aims at providing maximum robustness to this kind of modeling errors and disturbances, and this is succeeded through the appropriate selection of the attenuation coefficient $\rho$ which appears in the associated Riccati equation. Actually, the minimum value of $\rho$ for which there exists a solution for the algebraic Riccati equation (in the form of a positive definite symmetric matrix $P$ ) is the one that provides the control loop with maximum robustness. The above have been explained in "The Role of Riccati Equation Coefficients in $H_{\infty}$ Control Robustness" section of manuscript.

Remark 2 The control method that is presented in the article and which is based on nonlinear H-infinity control theory can be compared against global linearization methods, e.g., those based on differential flatness theory and Lyapunovbased methods (used by adaptive control schemes) [16-18]. As a general remark it can be stated that the nonlinear $\mathrm{H}$ infinity control, yet conceptually more simple than the other 
Fig. 3 a Plot of the $x-y$ trajectory followed by the mobile robot in case of the parallel parking maneuver, and b convergence of the robot's state variables $x_{1}=x, x_{2}=y$ and $x_{3}=\theta$ to the associated reference setpoints

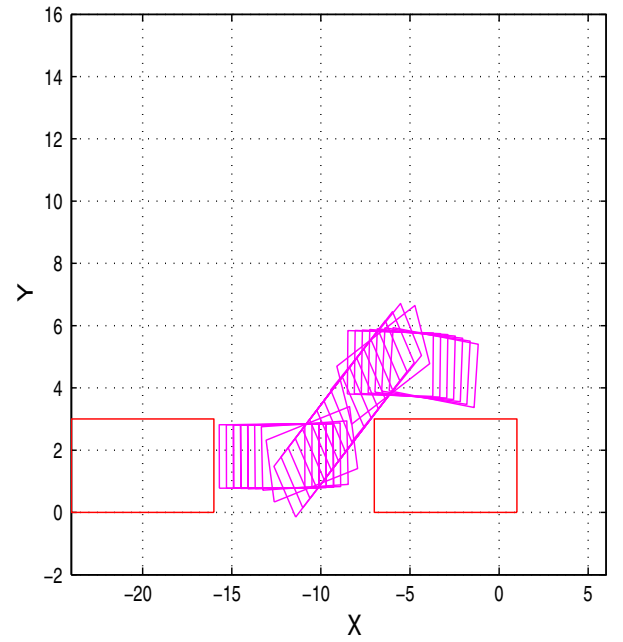

(a)
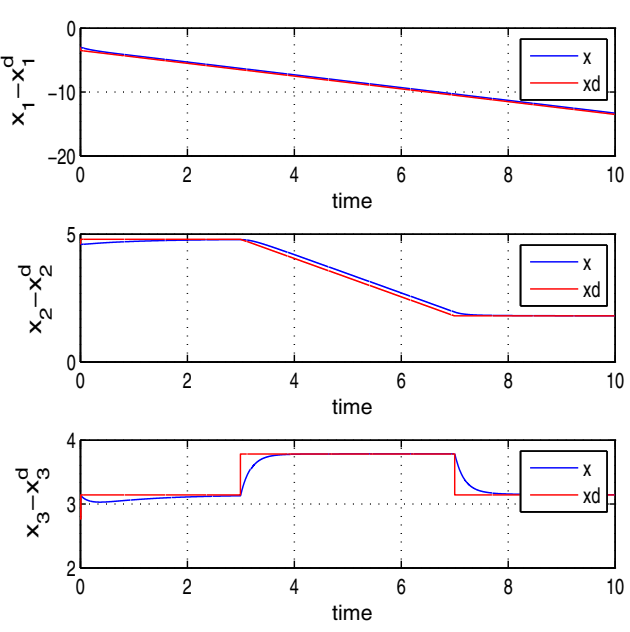

(b)
Table 1 Tracking RMSE in the disturbance-free case

\begin{tabular}{llll}
\hline & $R M S E_{x}$ & $R M S E_{y}$ & $R M S E_{\theta}$ \\
\hline Path $_{1}$ & $36.00 \times 10^{-4}$ & $39.00 \times 10^{-4}$ & $10.00 \times 10^{-4}$ \\
Path $_{2}$ & $7.90 \times 10^{-4}$ & $12.00 \times 10^{-4}$ & $2.40 \times 10^{-4}$ \\
\hline
\end{tabular}

Table 2 Tracking RMSE in motion under disturbances

\begin{tabular}{llll}
\hline & $R M S E_{x}$ & $R M S E_{y}$ & $R M S E_{\theta}$ \\
\hline Path $_{1}$ & $37.00 \times 10^{-4}$ & $54.00 \times 10^{-4}$ & $13.00 \times 10^{-4}$ \\
Path $_{2}$ & $14.00 \times 10^{-4}$ & $16.00 \times 10^{-4}$ & $2.41 \times 10^{-4}$ \\
\hline
\end{tabular}

two control approaches, is a reliable and efficient solution for the problem of autonomous vehicles' control. About the computational burden of the proposed control method, this is somehow elevated with respect to the control based on linearizing diffeomorphisms. This is because the proposed control method requires the solution of a Riccati equation at each step of the control algorithm followed by the computation of the gains of the feedback controller. Unlike this, methods based on diffeomorphisms proceed directly to the computation of the feedback control gains since the preceding linearization transformation is performed offline. However, the diffeomorphisms-based linearization can be cumbersome and not an easy to conceive procedure. Therefore, the benefit from avoiding a certain amount of computations in the diffeomorphisms-based control may be lost if the diffeomorphisms-stage is mathematically demanding.

\section{Conclusions}

A new nonlinear feedback control method has been developed based on approximate linearization and the use of $H_{\infty}$ control and stability theory. As a case study the problem of motion control of an autonomous robotic vehicle has been considered. It has been shown that the proposed control scheme enables the robot to track any type of reference path and to execute elaborated tasks such as parallel parking. The first stage of the proposed control method is the linearization of the robot's kinematic model using first order Taylor series expansion and the computation of the associated Jacobian matrices. The errors due to the approximative linearization have been considered as disturbances that affect, together with external perturbations, the robot's model.

At a second stage the implementation of $H_{\infty}$ feedback control has been proposed. Using the linearized model of the vehicle an $\mathrm{H}$-infinity feedback control law is computed at each iteration of the control algorithm, after previously solving an algebraic Riccati equation. The known robustness features of H-infinity control enable to compensate for the errors of the approximative linearization, as well as to eliminate the effects of external perturbations. The efficiency of the proposed control scheme is shown analytically and is confirmed through simulation experiments.

Comparing to other nonlinear control methods which are based on the exact linearization of the robot's model it can be stated that the proposed $H_{\infty}$ control uses the approximately linearized model of the robotic vehicle without implementing elaborated state transformations (diffeomorphisms) that finally bring the system to a linear form. Of course the computation of Jacobian matrices and the need to solve at each iteration of the algorithm a Riccati equation is also a computationally cumbersome procedure, especially for state-space models of large dimensionality. Moreover, this approximative linearization introduces additional perturbation terms which the $H_{\infty}$ controller has to eliminate. The continuous need for compensation of such cumulative linearization errors brings the $H_{\infty}$ controller closer to its robustness limits. 


\section{References}

1. Li, T.H.S., Yeh, Y.C., Wu, J.D., Hsiao, M.Y., Chen, C.Y.: Multifunctional intelligent autonomous parking controllers for carlike mobile robots. IEEE Trans. Ind. Electron. 57(5), 1687-1699 (2010)

2. Vepa, R.: Nonlinear unscented $H_{\infty}$ suspension and tracking control of mobile vehicles. IEEE Trans. Veh. Technol. 61(4), 1543-1553 (2012)

3. Rigatos, G., Siano, P.: An H-infinity feedback control approach to autonomous robot navigation. In: IEEE IECON 2014, Dallas, Texas, October 2014

4. Kanghyun, N., Fujimoto, M., Hori, Y.: Advanced motion control of electric vehicles based on robust lateral tire force control via active front steering. IEEE/ASME Trans. Mechatron. 19(1), 289299 (2014)

5. Li, T.H.S., Chang, S.J., Chen, Y.X.: Implementation of human-like driving skills by autonomous fuzzy behavior control on an FPGAbased car-like mobile robot. IEEE Trans. Ind. Electron. 50(5), 867880 (2003)

6. Hwang, C.L., Chang, L.J., Yu, Y.S.: Network-based fuzzy decentralized sliding-mode control for car-like mobile robots. IEEE Trans. Ind. Electron. 54(1), 574-585 (2007)

7. Wada, M., Yoon, K., Hashimoto, H.: Development of advanced parking assistance system. IEEE Trans. Ind. Electron. 50(1), 4-17 (2003)

8. Hwang, C.L., Shih, C.-Y.: A distributed active-vision networkspace approach for the navigation of a car-like wheeled robot. IEEE Trans. Ind. Electron. 56(3), 846-855 (2009)

9. Yamaguchi, Y., Murakami, T.: Adaptive control for virtual steering characteristics on electric vehicle using steer-by-wire system. IEEE Trans. Ind. Electron. 56(5), 1585-1594 (2009)
10. Chen, H., Ma, M.M., Wang, H., Liu, Z.Y., Cai, Z.X.: Moving horizon tracking $H_{\infty}$ control of wheeled mobile robots with actuator saturation. IEEE Trans. Control Syst. Technol. 17(2), 449-457 (2009)

11. Coelho, P., Nunes, U.: Path-following control of mobile robots in presence of uncertainties. IEEE Trans. Robot. 21(2), 252-261 (2005)

12. Sanchez-Solano, S., Cabrera, A.J., Baturone, I., Moreno-Velo, F.J., Brox, M.: FPGA implementation of embedded fuzzy controllers for robotic applications. IEEE Trans. Ind. Electron. 54(4), 1937-1945 (2007)

13. Baturone, I., Moreno-Velo, F.J., Blanco, V., Ferruz, J.: Design of embedded DSP-based fuzzy controllers for autonomous mobile robots. IEEE Trans. Ind. Electron. 55(2), 928-936 (2008)

14. Möller, B., Deutscher, J., Grodde, S.: Continuous curvature trajectory design and feedforward control for parking a car. IEEE Trans. Control Syst. Technol. 15(3), 541-553 (2007)

15. Lee, T.C., Tsai, C.Y., Song, K.T.: Fast parking control of mobile robots: a motion planning approach with experimental validation. IEEE Trans. Control Syst. Technol. 12(5), 661-676 (2004)

16. Rigatos, G.: Modelling and Control for Intelligent Industrial Systems: Adaptive Algorithms in Robotics and Industrial Engineering. Springer, Berlin (2011)

17. Rigatos, G.: Advanced Models of Neural Networks: Nonlinear Dynamics and Stochasticity in Biological Neurons. Springer, Dordrecht (2013)

18. Rigatos, G.: Nonlinear Control and Filtering Using Differential Flatness Theory Approaches: Applications to Electromechanical Systems. Springer, Switzerland (2015) 